\title{
Inhibition of microglial activation by minocycline reduced preoligodendrocyte injury in a neonatal rat brain slice model
}

\author{
Junrong Huang, MD, Gang Liu, MD, Bowen Shi, MD, Guochen Shi, MD, Xiaomin He, MD, PhD, \\ Zhaohui Lu, MD, PhD, Jinghao Zheng, MD, PhD, Haibo Zhang, MD, PhD, Huiwen Chen, MD, PhD, and \\ Zhongqun $\mathrm{Zhu}, \mathrm{MD}, \mathrm{PhD}$
}

\section{ABSTRACT}

Background: Periventricular leukomalacia is a common white-matter injury after neonatal cardiac surgery; however, its potential cellular mechanism remains uncertain. There is limited study regarding periventricular leukomalacia treatment.

Methods: A neonatal rat brain slice perfusion model was used for reproducing the condition of cardiopulmonary bypass, and oxygen glucose deprivation simulated circulatory arrest. Seven-day-old Sprague-Dawley rats were randomly divided into 7 groups: (1) control group with $36^{\circ} \mathrm{C}$; (2) 60 minutes of oxygen glucose deprivation group on $15^{\circ} \mathrm{C}, 25^{\circ} \mathrm{C}, 36^{\circ} \mathrm{C}$, respectively; and (3) 60 minutes of oxygen glucose deprivation group on $15^{\circ} \mathrm{C}, 25^{\circ} \mathrm{C}, 36^{\circ} \mathrm{C}$, plus minocycline $(10 \mu \mathrm{mol} /$ $\mathrm{L})$, respectively. Immunohistochemistry, Western blot, and inflammatory mediators were compared after the perfusion procedures in the different groups.

Results: This neonatal rat brain slice perfusion with oxygen glucose deprivation model could replicate the pathophysiologic process and injury after cardiopulmonary bypass and hypothermic circulatory arrest. With the increase of oxygen glucose deprivation perfusion temperature, we found that both microglia activation and preoligodendrocyte loss increased. The application of minocycline can significantly inhibit microglial activation and preoligodendrocyte cells loss in the normothermic $\left(36^{\circ} \mathrm{C}\right)$ and moderate hypothermia $\left(25^{\circ} \mathrm{C}\right)$ oxygen glucose deprivation groups $(P<.05)$, with accompanying significant decreasing microglial inflammatory productions; however, no significant improvement was found in the deep hypothermia $\left(15^{\circ} \mathrm{C}\right)$ group.

Conclusions: The microglial activation may play a key role in preoligodendrocyte injury in the ex vivo neonatal rat brain slice perfusion and circulatory arrest model. Inhibition of microglial activation with minocycline may be an attractive target for white-matter protection during cardiopulmonary bypass and hypothermic circulatory arrest. (J Thorac Cardiovasc Surg 2018;156:2271-80)

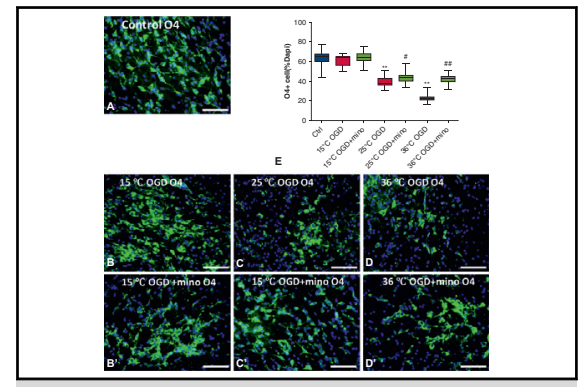

Survival of preOLs after OGD was improved by minocycline.

\section{Central Message}

Microglial activation may play a key role in preOL injury. Inhibition of microglial activation with minocycline may be an attractive target for WM protection during $\mathrm{CPB}$ and HCA.

\section{Perspective}

Cerebral WM injury is thought to contribute to the neurodevelopmental complications after CPB. Our findings demonstrate that microglial activation is an important cellular mechanism underlying preOL injury. Inhibition of microglial activation might be of potential value in terms of therapeutic intervention for attenuating WM injury after neonatal cardiac surgery.

See Editorial Commentary page 2281

\footnotetext{
From the Department of Cardiothoracic Surgery, Heart Center, Shanghai Children's Medical Center, Shanghai Jiaotong University School of Medicine, Shanghai, China.

Funding for this study was supported by Shanghai Hygiene Science Research (20114121), Research Project of Pudong New Area Health and Family Planning Commission (PW2017D-8), and the grant of Shanghai Science and Technology Commission (14411964900).

J.H. and G.L. contributed equally to this work.

Received for publication June 14, 2017; revisions received May 9, 2018; accepted for publication June 11, 2018; available ahead of print Aug 16, 2018.

Address for reprints: Zhongqun Zhu, MD, PhD, Department of Cardiothoracic Surgery, Heart Center, Shanghai Children's Medical Center, Shanghai Jiaotong University School of Medicine, 1678 Dongfang Rd, Shanghai, China (E-mail: zzqheart@aliyun.com).

$0022-5223 / \$ 36.00$

Copyright (c) 2018 Published by Elsevier Inc. on behalf of The American Association for Thoracic Surgery

https://doi.org/10.1016/j.jtcvs.2018.06.038
}

Periventricular leukomalacia (PVL) is a common complication after neonatal cardiac surgery and is associated with later neurologic dysfunction, including attention deficit, hyperactivity, impairment of working memory, and verbal dysfunction. ${ }^{1-3}$ PVL is characterized by both focal necrosis in the deep white matter (WM), with loss of

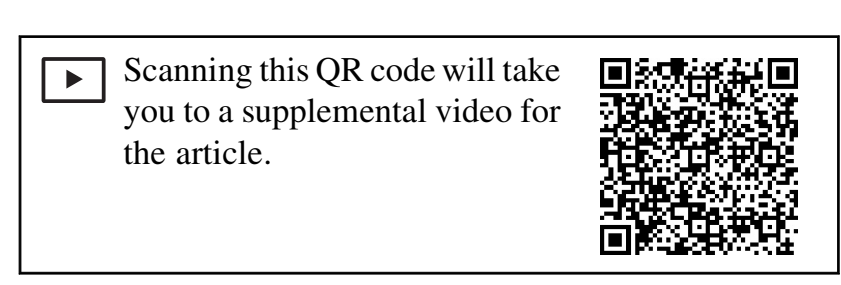




$$
\begin{aligned}
& \text { Abbreviations and Acronyms } \\
& \begin{aligned}
\text { aCSF } & =\text { artificial cerebrospinal fluid } \\
\text { CPB } & =\text { cardiopulmonary bypass } \\
\text { HCA } & =\text { hypothermic circulatory arrest } \\
\text { Iba1 } & \text { ionizing calcium-binding adaptor } \\
& \text { molecule } 1 \\
\text { IL } & =\text { interleukin } \\
\text { MBP } & \text { myelin basic protein } \\
\text { OGD } & =\text { oxygen glucose deprivation } \\
\text { PBS } & =\text { phosphate-buffered saline } \\
\text { preOL } & =\text { preoligodendrocyte } \\
\text { PVL } & =\text { periventricular leukomalacia } \\
\text { TNF- } \alpha=\text { tumor necrosis factor alpha } & \text { WM }=\text { white matter }
\end{aligned}
\end{aligned}
$$

all cellular elements, and diffuse injury to preoligodendrocytes (preOLs) or axons in the central cerebral WM. ${ }^{4,5}$ Diffuse WM injury is the predominant neuropathologic lesion in infants with congenital heart defects, which is followed by hypomyelination, caused by a loss of preOLs and mature myelin-producing oligodendrocytes. ${ }^{2-6}$ Microglia are the resident immune cells in the brain and have emerged as a potential convergence point in the potentiation of WM injury from the deleterious effects of inflammation and neurotoxicity in PVL. ${ }^{5,7,8}$ Although cardiopulmonary bypass (CPB) and hypothermic circulatory arrest (HCA) are regarded to be significant risk factors for PVL, the underlying cellular mechanism remains unclear.

Recent studies have identified diffuse WM injury and microglial activation in CPB models. ${ }^{6,9}$ However, it is not clear whether inhibition of microglial activation can reduce the loss of preOLs after CPB and HCA. As a semisynthetic second-generation tetracycline, minocycline has been shown to provide protection against ischemic brain injury and neuronal degenerative diseases through inhibition of microglial activation. ${ }^{10,11}$ In the present study, we aimed to determine the underlying role of microglial activation in preOL injury and provide further evidence that minocycline exerts protective effects through inhibition of microglial activation to alleviate preOL injury.

\section{MATERIALS AND METHODS \\ Animals and Experimental Procedure}

Postnatal day 7 Sprague-Dawley neonatal rat pups of both sexes were used for brain slice preparation. Brain maturation is delayed by approximately 1 month in neonates with complex congenital heart defects. ${ }^{12}$ Postnatal day 7 Sprague-Dawley rats were used as a model to investigate WM injury because they have a maturation level similar to the human fetus at 30 to 36 weeks' gestation. ${ }^{13,14}$ All animal experiments were approved by the Ethics Committee for Laboratory Animals, Shanghai Institute of Materia Medica, Chinese Academy of Sciences (Shanghai, China). The brains were quickly dissected from postnatal day 7 rats and immediately placed

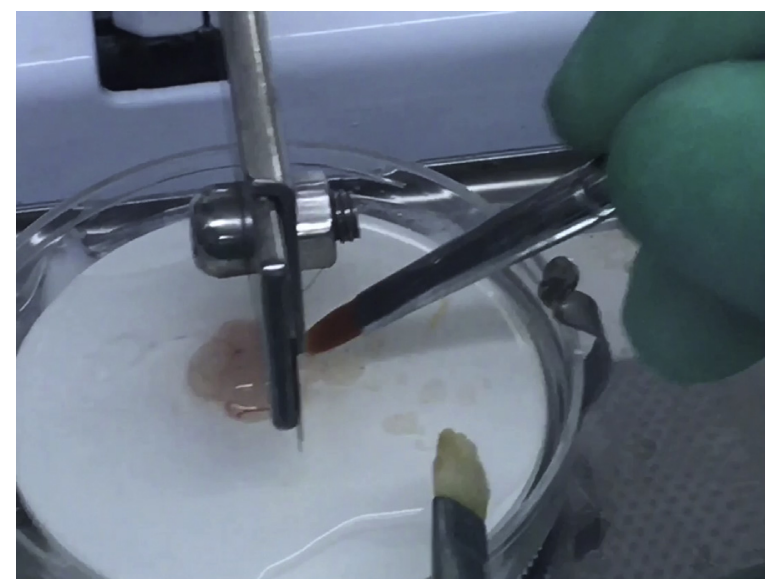

VIDEO 1. Brain slice model. The brains were quickly dissected from postnatal day 7 rats and immediately placed in ice-cold aCSF. Coronal sections (400- $\mu \mathrm{m}$ thick) were cut using a Mcllwain tissue chopper (Mickle Laboratory Engineering Co, Surrey, UK). Video available at: https:// www.jtcvs.org/article/S0022-5223(18)31783-5/fulltext.

in ice-cold artificial cerebrospinal fluid (aCSF) containing (in mmol/L) $125 \mathrm{NaCl}, 2.5 \mathrm{KCl}, 1 \mathrm{MgCl} 2,1.25 \mathrm{NaH}_{2} \mathrm{PO}_{4}, 2 \mathrm{CaCl}_{2}, 25 \mathrm{NaHCO}_{3}, 25$ glucose, 75 sucrose, equilibrated with $95 \% \mathrm{O}_{2} / 5 \% \mathrm{CO}_{2}$, $\mathrm{pH}$ 7.4. Coronal sections (400- $\mu \mathrm{m}$ thick) were cut using a Mcllwain tissue chopper (Mickle Laboratory Engineering Co, Surrey, UK) (Video 1, brain slice model). Only slices containing corpus callosum tissue, which is a major structure in the WM of the mouse brain, were included in perfusion experiments ( 3 per animal). Slices were placed in a holding chamber containing oxygenated aCSF to recover at $25^{\circ} \mathrm{C}$ for 1 to 2 hours after slicing. The perfusate temperature was then increased to $37^{\circ} \mathrm{C}$ at least 1 hour before continuing with the experimental protocol. A total of 4 to 8 slices from 2 to 3 littermates were then transferred to a customized tissue chamber and perfused (1 $\mathrm{mL} / \mathrm{min}$ ) with glucose containing oxygenated aCSF containing (in mmol/L) $125 \mathrm{NaCl}, 2.5 \mathrm{KCl}, 1 \mathrm{MgCl}_{2}, 1.25 \mathrm{NaH}_{2} \mathrm{PO}_{4}, 2 \mathrm{CaCl}_{2}, 25$ $\mathrm{NaHCO}_{3}, 10$ glucose, equilibrated with $95 \% \mathrm{O}_{2} / 5 \% \mathrm{CO}_{2}, \mathrm{pH} 7.4$. Each chamber consisted of 2 compartments with parallel perfusion circuits, enabling simultaneous comparison of 2 perfusion conditions. A perfusion system and chamber were placed in an incubator (Shanghai Boxun Industry and Commerce CO, Medical Equipment Factory, Shanghai, China) to control the temperature during the experiment (Figure 1).

\section{Oxygen Glucose Deprivation}

Oxygen glucose deprivation (OGD) was used to replicate HCA during cardiac surgery. It was induced by changing the perfusion solution in which sucrose was substituted for glucose and which had been saturated with $95 \%$ $\mathrm{N}_{2} / 5 \% \mathrm{CO}_{2}$. After OGD, the slices were reperfused with glucose containing aCSF saturated with $95 \% \mathrm{O}_{2} / 5 \% \mathrm{CO}_{2}$ mixture and varying lengths of recovery to allow ischemia-reperfusion/reoxygenation to the brain slices.

\section{Perfusion Protocol}

To simulate the $\mathrm{CPB}$ strategies under different temperatures, we subdivided the models into normothermic $\left(36^{\circ} \mathrm{C}\right)$, moderate hypothermia $\left(25^{\circ} \mathrm{C}\right)$, and deep HCA $\left(15^{\circ} \mathrm{C}\right)$ groups. Brain slices were assigned to 1 of 7 groups: control at $36^{\circ} \mathrm{C}$; OGD for 60 minutes at $15^{\circ} \mathrm{C}, 25^{\circ} \mathrm{C}$, and $36^{\circ} \mathrm{C}$; and $\mathrm{OGD}$ for 60 minutes at $15^{\circ} \mathrm{C}, 25^{\circ} \mathrm{C}$, and $36^{\circ} \mathrm{C}$, plus minocycline. Before OGD, the slices were first cooled to the target temperature according to group for 30 minutes. OGD was then performed for 60 minutes as described. To replicate HCA-induced hypothermic ischemia-reperfusion/ reoxygenation, the slices were reperfused with glucose containing oxygenated aCSF for 10 minutes, followed by a 30 -minute rewarming period, 


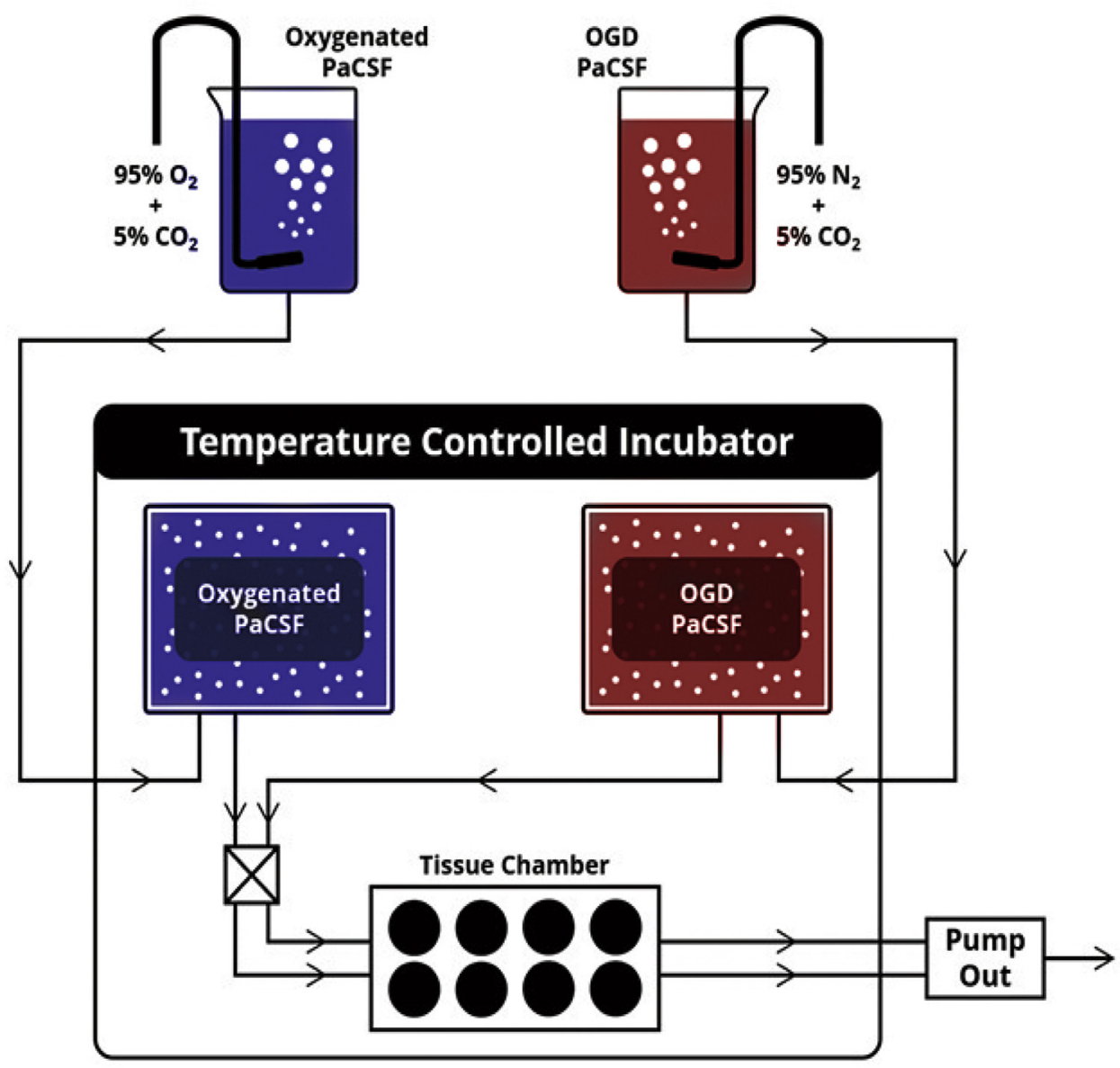

FIGURE 1. Schematic diagram of ex vivo brain slice perfusion model. PaCSF, Protective modified artificial cerebrospinal fluid; $O G D$, oxygen glucose deprivation.

followed by continued perfusion for 24 hours (Video 2, perfusion system). The control group was perfused with glucose containing oxygenated aCSF at $36^{\circ} \mathrm{C}$ for the same period without OGD. Slices in the minocycline experiments were preincubated with $10 \mu \mathrm{mol} / \mathrm{L}$ minocycline ${ }^{15}$ for 1 hour and subjected to the perfusion solution according to the perfusion protocol (Figure 2).

\section{Immunohistochemistry}

After the perfusion procedures, slices were fixed in $4 \%$ paraformaldehyde in phosphate-buffered saline (PBS) for 1 hour at room temperature and then cryoprotected by incubating in $30 \%$ sucrose at $4{ }^{\circ} \mathrm{C}$ overnight for 48 hours. Brain slices from each group were sectioned into $20-\mu \mathrm{m}$ slices using a cryostat. Sections from the outer $80 \mu \mathrm{m}$ of each slice were excluded to avoid tissue that may have been damaged during slice preparation. For immunofluorescence staining, sections were permeabilized using $0.5 \%$ Triton X-100 for 1 hour at room temperature and then blocked for 1 hour using $1 \%$ bovine serum albumin, with $10 \%$ goat serum in PBS. Subsequently, sections were incubated with anti-O4 (1:100; Millipore Corporation, Burlington, Mass), anti-myelin basic protein (MBP) (1:200; Abcam, San Francisco, Calif), or anti-IBa-1 (1:200; Wako, Japan) diluted in blocking buffer at $4^{\circ} \mathrm{C}$ overnight. Sections were washed 3 times with PBS and incubated with Alexa Fluor 488-conjugated goat antimouse immunoglobulin (Ig) G, IgM (1:200; Invitrogen, Carlsbad, Calif), Alexa Fluor 488-conjugated goat anti-rabbit IgG (1:200; Invitrogen), or Alexa Fluor 555-conjugated goat anti-rabbit IgG (1:200; Invitrogen) for 1 hour at room temperature. Positive cells were counted using fluorescence microscopy. Three randomly chosen high-power fields (magnification, $400 \times$ ) in the corpus callosum of each section were selected for assessment. Positive cell staining was quantified using Image-Pro Plus (Media Cybernetics, Inc, Shanghai, China).

\section{Western Blot}

Brain tissues were minced and homogenized in ice-cold RIPA lysis buffer, followed by centrifugation at $12,000 \mathrm{rpm}$ for 5 minutes at $4^{\circ} \mathrm{C}$ to remove debris. Based on the concentration measured using the bicinchoninic acid assay, equal amounts of ionizing calcium-binding adaptor molecule 1 (Iba1) and beta-actin per lane were subjected to a $10 \%$ sodium dodecyl-polyacrylamide gel electrophoresis for separation and transferred onto a polyvinylidene difluoride membrane. Target blots were blocked with $5 \%$ nonfat milk for 1 hour at room temperature and incubated overnight at $4^{\circ} \mathrm{C}$ using specific antibodies against Iba1 and beta-actin. The membranes were then incubated for 1 hour at room temperature with the corresponding secondary antibodies after washing with TBST 3 times. Enhanced chemiluminescence detection reagents and a gel imaging system (Tanon Science \& Technology Co, Ltd, Shanghai, China) were used to visualize the protein bands on the membranes.

\section{Measurement of Tumor Necrosis Factor-Alpha and Interleukin-6}

Measurements of tumor necrosis factor alpha (TNF- $\alpha)$ and interleukin (IL)-6 in rat brain slices were performed using a commercially available enzyme-linked immunoassay kit according to the manufacturer's instructions. 


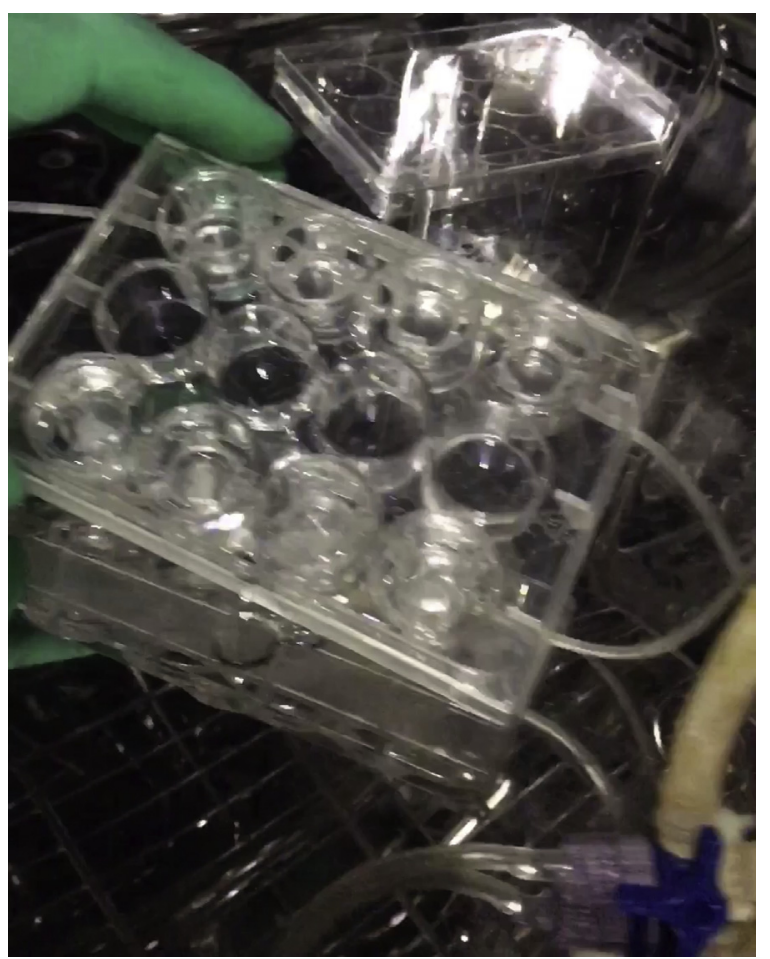

VIDEO 2. Perfusion system. The slices were first cooled to the target temperature according to group for 30 minutes. To replicate HCA-induced hypothermic ischemia-reperfusion/reoxygenation, the slices were reperfused with glucose containing oxygenated aCSF for 10 minutes, followed by a 30 -minute rewarming period, followed by continued perfusion for 24 hours. The control group was perfused with glucose containing oxygenated aCSF at $36^{\circ} \mathrm{C}$ for the same period without OGD. Slices in the minocycline experiments were preincubated with $10 \mu \mathrm{mol} / \mathrm{L}$ minocycline for 1 hour. Video available at: https://www.jtcvs.org/article/S0022-5223(18)31783-5/ fulltext.

\section{Statistical Analysis}

Data were expressed as mean \pm standard deviation and assessed using 1-way analysis of variance, followed by a Bonferroni post hoc test using InStat (GraphPad Software Inc, La Jolla, Calif). Data were collected from at least 3 separate experiments.

\section{RESULTS}

Myelin Basic Protein Immunohistochemistry of Ex Vivo Brain Slices in the Oxygen Glucose Deprivation Perfusion Model

Changes in MBP expression can be used as an index of the degree of WM injury. To estimate the efficiency of the model to replicate $\mathrm{CPB} / \mathrm{HCA}$, the expression of
MBP after the perfusion protocol was tested. There was no significant difference in immunofluorescence or morphologic changes in MBP-positive cells between the control group and the $15^{\circ} \mathrm{C}$ OGD group. However, MBP-positive cells at $25^{\circ} \mathrm{C}$ and $36^{\circ} \mathrm{C}$ in the OGD group exhibited different degrees of morphologic changes and decreased fluorescence, which was significantly correlated with OGD temperature (Figure 3). These results exhibited a pattern similar to the pathophysiology of WM injury associated with $\mathrm{CPB} / \mathrm{HCA}$ in other clinical and laboratory studies. ${ }^{2,5}$ Rapid reduction in MBP expression demonstrated that MBP was sensitive to OGD, which instigated acute MBP degradation. This finding demonstrated that this model can effectively replicate WM injury induced by $\mathrm{CPB} / \mathrm{HCA}$ in vitro.

\section{Minocycline Improves the Survival of Preoligodendrocytes After Oxygen Glucose Deprivation in an Ex Vivo Brain Slice Model}

Damage to preOLs caused by energy failure after hypoxia/ischemia or OGD has been characterized by excitotoxic cell death and impaired maturation. OGD was applied for 1 hour in an in ex vivo brain slice model, and the numbers of preOLs after OGD with and without minocycline after the total perfusion time were analyzed. Positive $\mathrm{O} 4$ cells were expressed as a percentage of all $4^{\prime}, 6$-diamidino-2phenylindole-positive cells. The number of preOLs under control conditions was significantly decreased after $25^{\circ} \mathrm{C}$ and $36^{\circ} \mathrm{C}$ OGD without minocycline. This decrease was prevented by $10 \mu \mathrm{mol} / \mathrm{L}$ minocycline $\left(36^{\circ} \mathrm{C}\right.$ OGD vs $36^{\circ} \mathrm{C}$ OGD $+10 \mu \mathrm{mol} / \mathrm{L}$ minocycline $[P<.01]$; and $25^{\circ} \mathrm{C}$ OGD vs $25^{\circ} \mathrm{C}$ OGD $+10 \mu \mathrm{mol} / \mathrm{L}$ minocycline $[P<.05]$ ) (Figure 4 and Table 1). These data demonstrated that OGD significantly affected preOLs and that minocycline could directly improve the survival of these cells after the insult.

\section{Minocycline Inhibits the Activation of Microglia After Oxygen Glucose Deprivation in an Ex Vivo Brain Slice Model}

Microglial activation was stimulated by OGD, which may produce cytotoxic effects in the brain slice model. Positive Iba1 cells were expressed as a percentage of all 4',6diamidino-2-phenylindole-positive cells, as indicated by an increase in cell size, irregular shape, thickened and

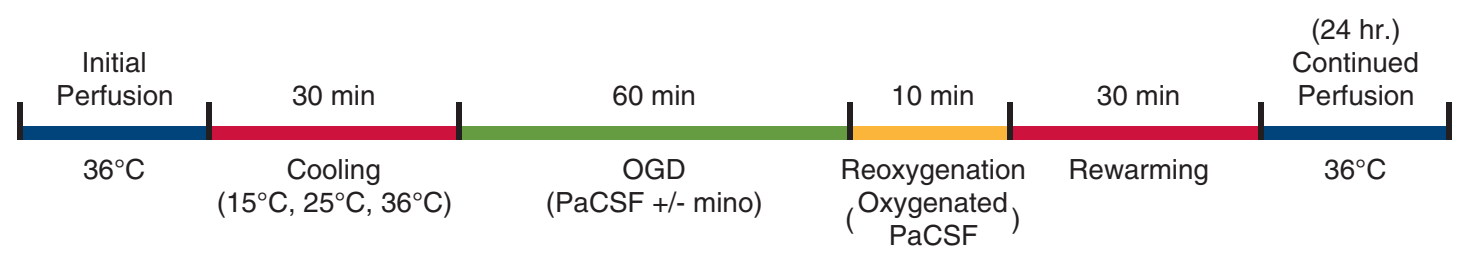

FIGURE 2. Schematic diagram of perfusion protocol. $O G D$, Oxygen glucose deprivation; $P a C S F$, protective modified artificial cerebrospinal fluid. 

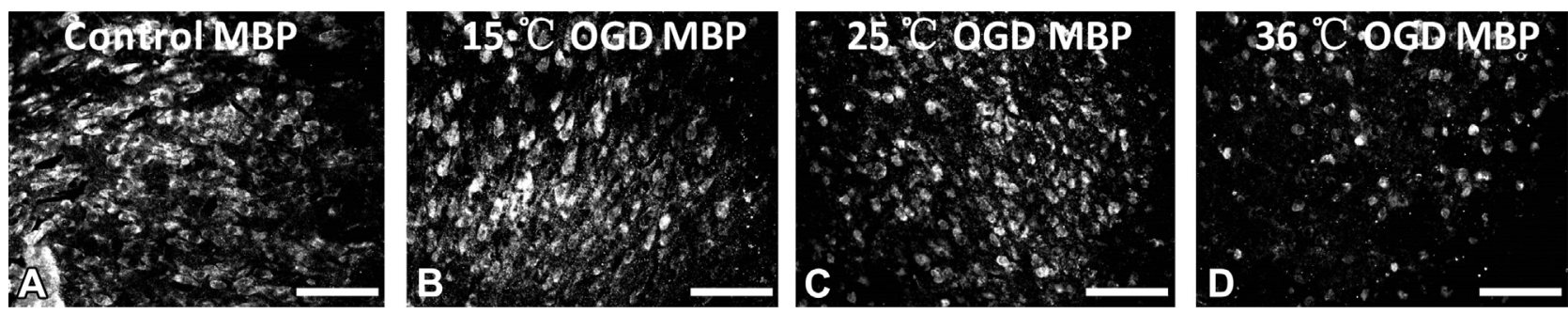

FIGURE 3. MBP immunohistochemistry of ex vivo brain slice OGD perfusion model. Immunostaining of MBP-positive labeled oligodendrocyte in postnatal day 7 rat brain tissue sections. White staining represents MBP-positive oligodendrocyte. Control group (A); OGD group: $15^{\circ} \mathrm{C}, 25^{\circ} \mathrm{C}$, and $36^{\circ} \mathrm{C}(\mathrm{B}, \mathrm{C}$, and D, respectively). Scale bar $=50 \mu \mathrm{m}$. $M B P$, Myelin basic protein; $O G D$, oxygen glucose deprivation.

shortened processes, and intensified Iba1 immunostaining density in the coronal section. Microglial activation was significantly increased after $36^{\circ} \mathrm{C}$ OGD without minocycline. This was inhibited by $10 \mu \mathrm{mol} / \mathrm{L}$ minocycline $\left(36^{\circ} \mathrm{C}\right.$ OGD vs $36^{\circ} \mathrm{C}$ OGD $+10 \mu \mathrm{mol} / \mathrm{L}$ minocycline $P<.01] ; 25^{\circ} \mathrm{C}$ OGD vs $25^{\circ} \mathrm{C}$ OGD $+10 \mu \mathrm{mol} / \mathrm{L}$
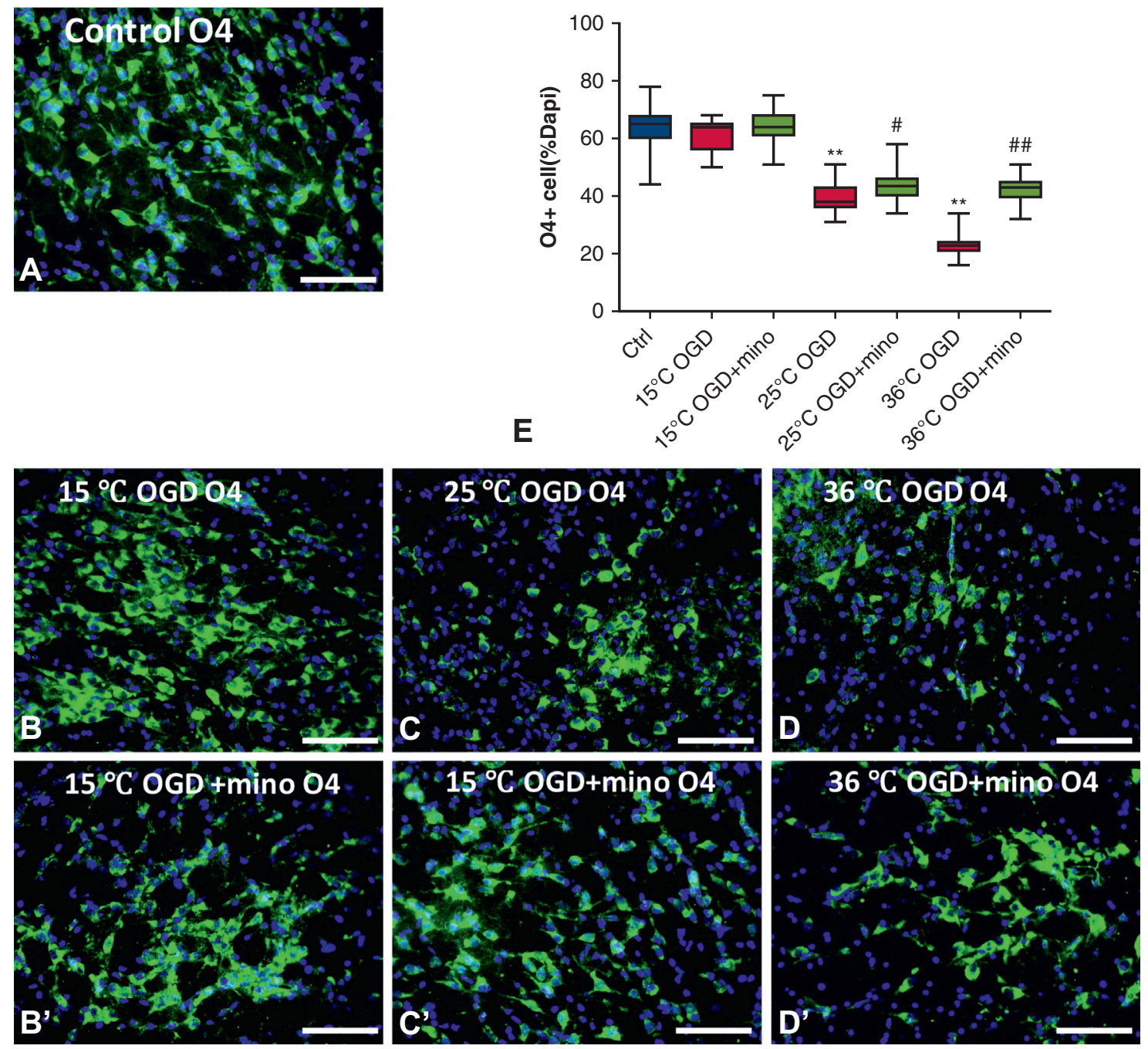

FIGURE 4. Survival of preOLs after OGD was improved by minocycline. Immunostaining of O4-positive labeled preOLs in postnatal day 7 rat brain tissue sections. Green staining represents O4-positive preOLs. Blue staining represents $4^{\prime}, 6$-diamidino-2-phenylindole. Control group (A); OGD group: $15^{\circ} \mathrm{C}$, $25^{\circ} \mathrm{C}$, and $36^{\circ} \mathrm{C}\left(\mathrm{B}, \mathrm{C}\right.$, and $\mathrm{D}$, respectively); minocycline-treated group: $15^{\circ} \mathrm{C}, 25^{\circ} \mathrm{C}$, and $36^{\circ} \mathrm{C}\left(\mathrm{B}^{\prime}, \mathrm{C}^{\prime}\right.$, and $\mathrm{D}^{\prime}$, respectively); $\mathrm{E}$, Coordinate graph. $* * P<.01$ control versus $25^{\circ} \mathrm{C}$ OGD; $* * P<.01$ control versus $36^{\circ} \mathrm{C}$ OGD; \#P<.05 $25^{\circ} \mathrm{C}$ OGD versus $25^{\circ} \mathrm{C}$ OGD + minocycline; \#\#P<.0136 $36^{\circ} \mathrm{C}$ OGD versus $36^{\circ} \mathrm{C} \mathrm{OGD}+$ minocycline. Data are expressed as box-and-whiskers plots of 3 independent experiments ( 3 sections/brain $\times 8$ rats/group). Scale bar $=50 \mu \mathrm{m}$. OGD, Oxygen glucose deprivation; mino, minocycline. 
TABLE 1. Comparison of preoligodendrocyte injury, microglial activation, and cytokines

\begin{tabular}{|c|c|c|c|c|c|c|c|c|c|c|c|c|c|}
\hline \multirow[b]{2}{*}{ Parameter } & \multirow[b]{2}{*}{ Control } & \multirow{2}{*}{$15^{\circ} \mathrm{C}$ OGD } & \multirow{2}{*}{$\begin{array}{c}15^{\circ} \mathrm{C} \text { OGD + } \\
\text { minocycline }\end{array}$} & \multirow[b]{2}{*}{$25^{\circ} \mathrm{C}$ OGD } & \multirow{2}{*}{$\begin{array}{c}25^{\circ} \mathrm{C} \text { OGD }+ \\
\text { minocycline }\end{array}$} & \multirow[b]{2}{*}{$36^{\circ} \mathrm{C}$ OGD } & \multirow{2}{*}{$\begin{array}{c}36^{\circ} \mathrm{C} \text { OGD + } \\
\text { minocycline }\end{array}$} & \multicolumn{6}{|c|}{$P$ value } \\
\hline & & & & & & & & 1 & 2 & 3 & 4 & 5 & 6 \\
\hline $\begin{array}{l}04+\text { cell } \\
\quad(\% \text { DAPI })\end{array}$ & $63.5 \pm 7.1$ & $61.6 \pm 5.3$ & $63.5 \pm 4.9$ & $30.6 \pm 4.9$ & $41.5 \pm 3.8$ & $22.8 \pm 3.4$ & $42.1 \pm 4.6$ & .077 & .084 & .003 & .037 & $<.001$ & .002 \\
\hline $\begin{array}{l}\text { Iba } 1+\text { cell } \\
\quad(\% \text { DAPI })\end{array}$ & $20.6 \pm 1.9$ & $16.8 \pm 3.9$ & $14.0 \pm 1.1$ & $27.6 \pm 6.7$ & $24.7 \pm 1.5$ & $36.5 \pm 8.1$ & $32.3 \pm 7.2$ & .039 & .073 & .006 & .008 & $<.001$ & .005 \\
\hline $\begin{array}{l}\text { Iba- } 1 / \beta \text {-actin } \\
\text { protein } \\
\text { expression }\end{array}$ & $0.25 \pm 0.03$ & $0.24 \pm 0.02$ & $0.22 \pm 0.01$ & $0.44 \pm 0.04$ & $0.41 \pm 0.15$ & $0.73 \pm 0.27$ & $0.66 \pm 0.24$ & .946 & .671 & $<.001$ & .039 & $<.001$ & .006 \\
\hline IL-6 (ng/L) & $3.7 \pm 0.5$ & $3.6 \pm 0.3$ & $3.4 \pm 0.4$ & $5.1 \pm 0.4$ & $4.0 \pm 0.2$ & $14.3 \pm 1.8$ & $9.9 \pm 1.4$ & .248 & .465 & .041 & .037 & .003 & .007 \\
\hline $\mathrm{TNF} \alpha(\mathrm{ng} / \mathrm{L})$ & $2.3 \pm 0.1$ & $2.5 \pm 0.2$ & $2.4 \pm 0.2$ & $5.3 \pm 0.7$ & $4.0 \pm 1.6$ & $8.8 \pm 3.9$ & $6.5 \pm 2.3$ & .547 & .850 & .038 & .045 & .006 & .009 \\
\hline
\end{tabular}

Data are expressed as mean \pm standard deviation by analysis of variance with Bonferroni comparisons. DAPI, 4',6-Diamidino-2-phenylindole; Ibal, ionizing calcium-binding adaptor molecule $1 ; I L$, interleukin 6 ; $O G D$, oxygen glucose deprivation; 1 , control versus $15^{\circ} \mathrm{C} \mathrm{OGD;}, 15^{\circ} \mathrm{C}$ OGD versus $15^{\circ} \mathrm{C}$ OGD + minocycline; 3 , control versus $25^{\circ} \mathrm{C}$ OGD; $4,25^{\circ} \mathrm{C}$ OGD versus $25^{\circ} \mathrm{C}$ OGD + minocycline; 5 , control versus $36^{\circ} \mathrm{C}$ OGD; $6,36^{\circ} \mathrm{C}$ OGD versus $36^{\circ} \mathrm{C}$ OGD + minocycline; $T N F \alpha$, tumor necrosis factor alpha.

minocycline $[P<.01]$ ) (Figure 5 and Table 1). Results of Iba1 protein expression after OGD in Western blot analysis also yielded the same results (Figure 6 and Table 1).

\section{Minocycline Decreases the Concentration Tumor Necrosis Factor- $\alpha$ and Interleukin-6 in an Ex Vivo Brain Slice Model}

The concentrations of TNF- $\alpha$ and IL- 6 were assayed in the different groups using enzyme-linked immunoassay (Figure 7 and Table 1). The levels of both TNF- $\alpha$ and IL6 were significantly increased at $25^{\circ} \mathrm{C}$ OGD $(P<.05)$ and $36^{\circ} \mathrm{C}$ OGD $(P<.01)$ compared with the control group, whereas it was downregulated with minocycline $(10 \mu \mathrm{mol} / \mathrm{L})$ compared with the $25^{\circ} \mathrm{C}$ OGD $(P<.05)$ and $36^{\circ} \mathrm{C}$ OGD groups $(P<.01)$.

\section{DISCUSSION}

In the present study, we identified preOL loss and acute axonal injuries in neonatal WM using a brain slice perfusion model consistent with previous studies. ${ }^{14,16} \mathrm{We}$ demonstrated that microglia were activated under conditions of OGD, which induced an increase in cytokine levels-TNF- $\alpha$ and IL6 in particular-which significantly exacerbated preOL injury. Inhibition of microglial activation with minocycline can reduce damage to preOLs secondary to CPB and circulatory arrest.

Given that a rodent brain slice model can be established to simulate the conditions of CPB and HCA, it has been widely used to develop pharmacologic therapies for neurologic disorders. ${ }^{14,16-18}$ Specific pathophysiologic brain injuries after $\mathrm{CPB}$, as well as HCA-induced inflammatory response and ischemia-reperfusion/reoxygenation, are consistent with the results of HCA-induced brain injury in large animal models. ${ }^{14,16,18}$ Similar results were obtained in this study (Figure 3). We found a rapid reduction in MBP levels 24 hours after an OGD insult, which was not consistent with a study that reported that decreases in
MBP expression in an in vivo model took 2 to 4 weeks. ${ }^{19}$ This can be explained by the fact that axonal injury was more severe in vitro, without any protection from the blood-brain barrier and lack of adequate nutrient supplementation from the cerebral circulation. ${ }^{19,20}$

Injury to preOLs with subsequent hypomyelination is the major pathologic lesion in diffuse WM injury. ${ }^{4,5}$ The cellular mechanisms of preOL injury during CPB or HCA are multifactorial. First, preOLs are intrinsically vulnerable to injury during the peak period of PVL. ${ }^{5}$ The time window for the highest risk for PVL in the human brain is between 24 and 32 weeks' gestation, when the cerebral WM is predominantly populated by preOLs. ${ }^{4,21}$ PreOLs are most vulnerable to excitotoxicity, oxidative stress, hypoxia, and CPB-induced damage within the known oligodendrocyte developmental stages. ${ }^{6,22}$ We used postnatal day 7 Sprague-Dawley neonatal rats because their cerebral maturation is equivalent to the third trimester in humans. ${ }^{5}$ In this model, autonomous cell-intrinsic vulnerability factors with extrinsic injury occurring at a particular time and place were associated with a rapid loss of preOLs after normothermic and hypothermic ischemia-reperfusion/ reoxygenation injury. Second, the causal relationship between microglial activation and preOL injury was identified using preOLs in co-culture with microglia in an in vitro model in the previous study. ${ }^{8}$ As the first-line defense cells in the brain, microglia are rapidly activated by ischemia/reperfusion or reoxygenation, as well as inflammation induced by CPB and HCA. This induces the secretion of a range of proinflammatory factors, including reactive oxygen species, reactive nitrogen species, glutamate, inducible nitric oxide synthase, NADPH oxidase, and inflammatory cytokines, which can mediate preOLs cytotoxicity and apoptosis, either directly or through autocrine or paracrine microglial activation. ${ }^{21-25}$ The brain environment during $\mathrm{CPB}$ and HCA can be reproduced using this mouse brainslice model. In this study, microglial activation with early 

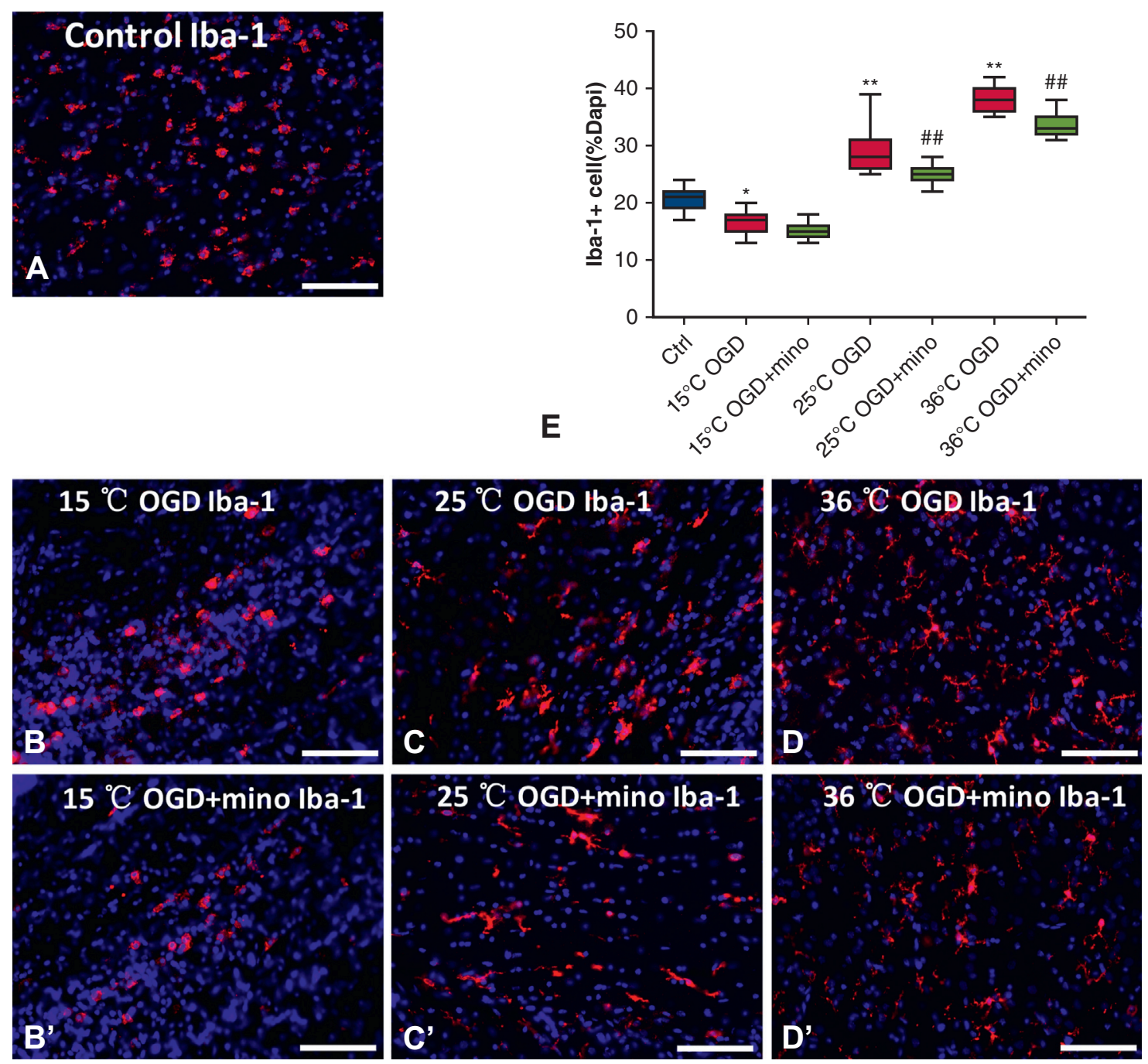

FIGURE 5. Activation of microglial cell after OGD was suppressed by minocycline. Immunostaining of Iba1-positive labeled microglial cell in postnatal day 7 rat brain tissue sections. Red staining represents Iba1-positive microglial cell. Blue staining represents $4^{\prime}, 6$-diamidino-2-phenylindole. Control group (A); OGD group: $15^{\circ} \mathrm{C}, 25^{\circ} \mathrm{C}$, and $36^{\circ} \mathrm{C}\left(\mathrm{B}, \mathrm{C}\right.$, and $\mathrm{D}$, respectively); minocycline-treated group: $15^{\circ} \mathrm{C}, 25^{\circ} \mathrm{C}$, and $36^{\circ} \mathrm{C}\left(\mathrm{B}^{\prime}, \mathrm{C}^{\prime}\right.$, and $\mathrm{D}^{\prime}$, respectively); $\mathrm{E}$, Coordinate graph. $* P<.05$ control versus $15^{\circ} \mathrm{C}$ OGD; $* * P<.01$ control versus $25^{\circ} \mathrm{C}$ OGD; $* * P<.01$ control versus $36^{\circ} \mathrm{C}$ OGD; \#\#P<.01 $25^{\circ} \mathrm{C}$ OGD versus $25^{\circ} \mathrm{C}$ OGD + minocycline; \#\#P<.0136 $36^{\circ} \mathrm{C}$ OGD versus $36^{\circ} \mathrm{C}$ OGD + minocycline. Data are expressed as box-and-whiskers plots of 3 independent experiments ( 3 sections/brain $\times 8$ rats/group). Scale bar $=50 \mu \mathrm{m}$. Ibal, Ionizing calcium-binding adaptor molecule 1; OGD, oxygen glucose deprivation; mino, minocycline.

increases in the number of Iba1-positive microglial cells and the release of TNF- $\alpha$ and IL6 with accompanying acute loss of preOLs were also found in the brain slice model (Figure 5). Prolonged microglial activation in the cerebral WM after normothermic and moderately hypothermic CPB has been observed. ${ }^{9}$ Thus, potentiation of these proinflammatory factors released from activated microglia significantly magnified ischemic or hypoxic injury to preOLs, which indicates that microglial activation may play a pivotal role in preOL injury. Third, astrocyte activation in protecting WM against hypothermic ischemiareperfusion/reoxygenation was identified, ${ }^{18}$ although we were unable to define astrocyte reactions in this study. The astrocyte reaction is altered after preoperative hypoxia and, thus, may contribute to increased WM injury secondary to injury associated with $\mathrm{CPB}$ and $\mathrm{HCA}$ in immature WM. ${ }^{18}$

Minocycline has been used to treat a variety of infectious diseases. ${ }^{11}$ Its neuroprotective properties have been identified in human neurodegenerative diseases, such as Parkinson's disease, Huntington's disease, and spinal cord injury, as well as neonatal WM injury. ${ }^{11}$ Anti-inflammatory actions, inhibition of matrix metalloproteinase, antiapoptotic and antinecrotic process, and 

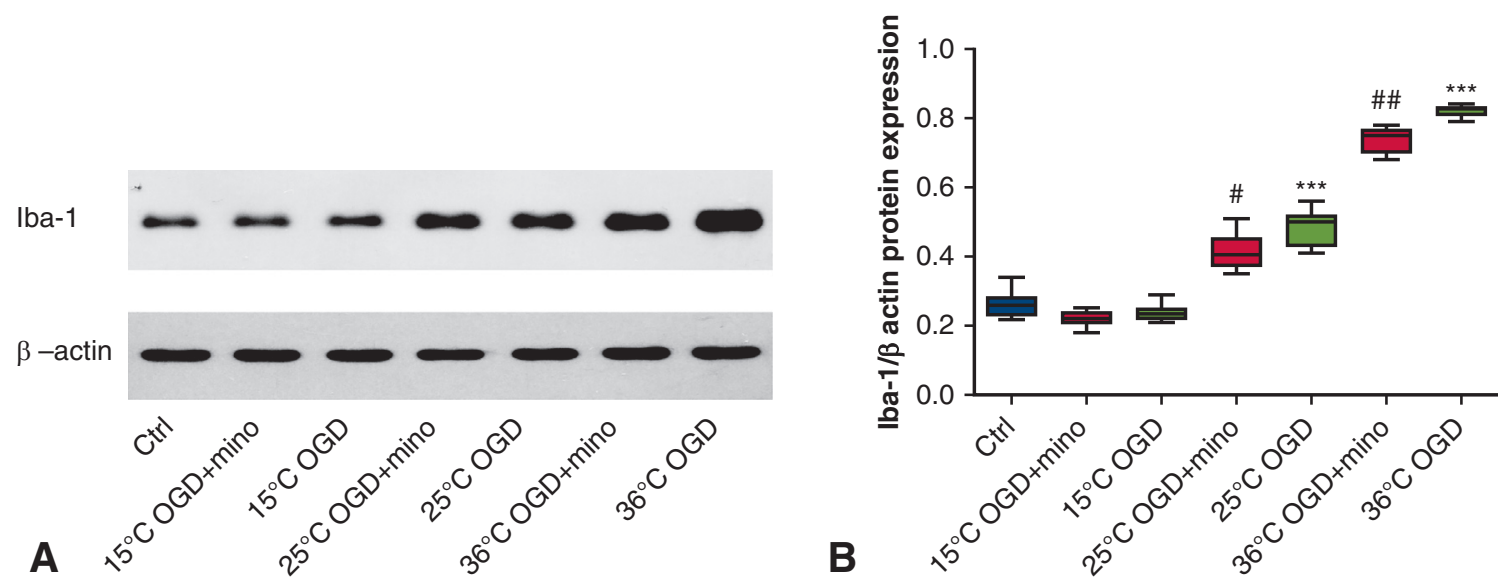

FIGURE 6. Western blot analysis of the effects of minocycline on Iba1 expression in rat brain slice after OGD. A, Western blot from rat brain slice tissue showed immunoreactivities for Iba1 and $\beta$-actin. B, Quantification of Western blot data. The graphs show the level of Iba1 expressed as a ratio of Iba1 and $\beta$-actin. ${ }^{* * *} P<.01$ control versus $25^{\circ} \mathrm{C}$ OGD; $* * * P<.01$ control versus $36^{\circ} \mathrm{C}$ OGD; $\# P<.0525^{\circ} \mathrm{C}$ OGD versus $25^{\circ} \mathrm{C}$ OGD + minocycline; \#\#P<.01 $36^{\circ} \mathrm{C}$ OGD versus $36^{\circ} \mathrm{C} \mathrm{OGD}+$ minocycline. Data are expressed as box-and-whiskers plots of 3 independent experiments ( 3 sections/brain $\times 8$ rats/group). Ibal, Ionizing calcium-binding adaptor molecule 1; $O G D$, oxygen glucose deprivation; mino, minocycline.

antioxidant action are some key processes involved in the neuroprotective mechanism of minocycline. The inflammatory process plays a critical role in microglia activation and accumulation, which contribute to the excessive production and release of proinflammatory cytokines. Thus, the anti-inflammatory actions of minocycline is a key mechanism in the inhibition of microglia activation. ${ }^{26}$ In the current study, minocycline significantly protected against preOL injury in the normothermic and moderately hypothermic groups (Figure 4). The protective effects of minocycline can be attributed, in large part, to inhibition of microglial activation (Figure 5). In addition, the direct protective actions of minocycline on oligodendroglial-lineage cells also suggested potential benefit in protecting against WM injury. ${ }^{15}$ Minocycline has been recognized as a potential neuroprotectant in neonatal models of brain injury. ${ }^{26}$ However, minocycline has demonstrated some side effects in neonates and infants, including stunted bone growth and bilirubin-induced brain damage by preventing the binding of bilirubin to albumin. The results of this study indicated that targeting the inhibition of microglial activation with the administration of minocycline into the priming fluid of $\mathrm{CPB}$ may have a potential role in improving neurologic outcomes after normothermic or moderately hypothermic CPB and HCA.
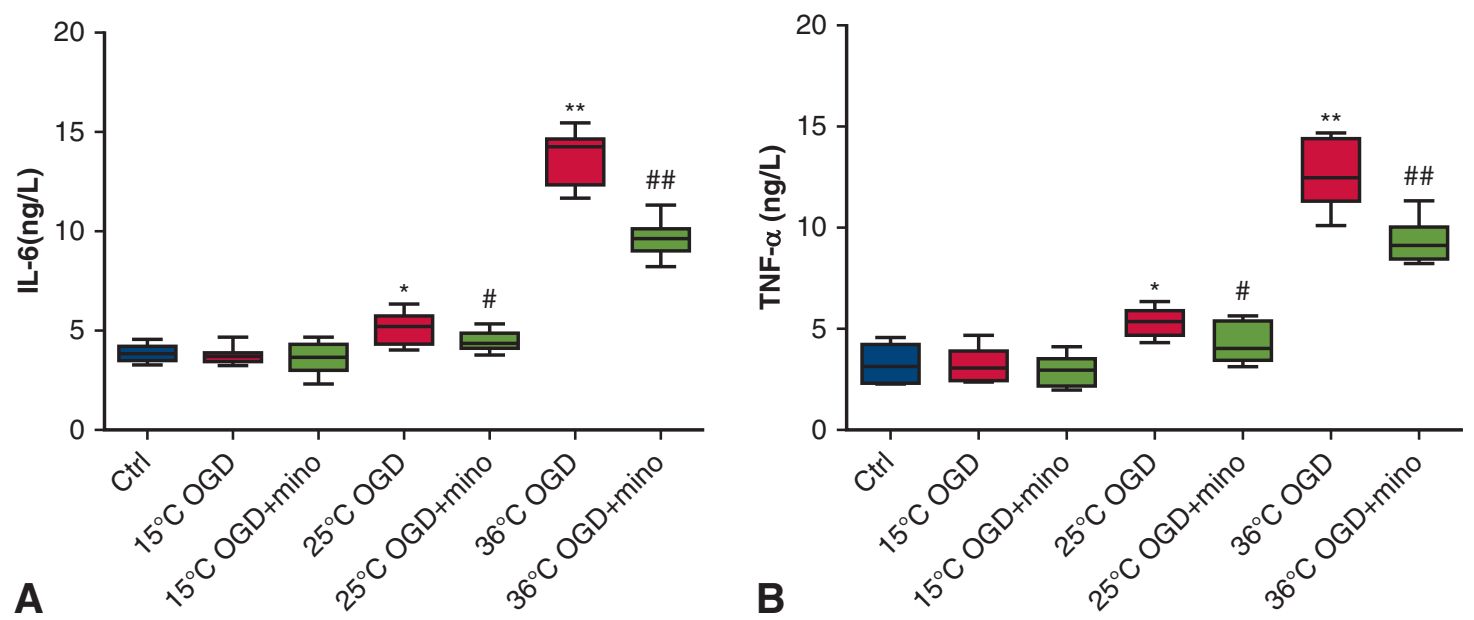

FIGURE 7. Enzyme-linked immunoassay analysis of TNF $\alpha$ and IL-6 concentration in ex vivo brain slice OGD perfusion model and minocycline treatment. A, Concentration of IL- 6 in each group. B, Concentration of TNF $\alpha$ in each group. $* P<.05$ control versus $25^{\circ} \mathrm{C}$ OGD; ** $P<.01$ control versus $36^{\circ} \mathrm{C}$ OGD; \#P<.05 $25^{\circ} \mathrm{C}$ OGD versus $25^{\circ} \mathrm{C}$ OGD + minocycline; \#\#P $<.0136^{\circ} \mathrm{C}$ OGD versus $36^{\circ} \mathrm{C}$ OGD + minocycline. Data are expressed as box-andwhiskers plots of 3 independent experiments ( 3 sections/brain $\times 8$ rats/group). IL, Interleukin; $O G D$, oxygen glucose deprivation; mino, minocycline; $T N F-\alpha$, tumor necrosis factor alpha. 
Although preOL protection was achieved in the normothermic and moderately hypothermic groups using minocycline, significant improvement in preOL injury was not observed in the deeply hypothermic $\left(15^{\circ} \mathrm{C}\right)$ group. This may be due to the significant pharmacokinetic and pharmacodynamic changes in deep hypothermia, which may have resulted in an adverse impact on the biodisposition and action of minocycline. ${ }^{27}$ Inhibition of microglia activation, which contributes to the limitation of inflammatory factors, can be found in deep hypothermia. ${ }^{10}$ Significant reduction in the expression of TNF- $\alpha$, IL-6, and Iba1-positive cells, and preOL loss 48 hours after OGD was also apparent in the isolated deep hypothermia group. However, deep hypothermia may also cause some extent of developmental arrest in the oligodendrocyte cell linage, which may eventually result in developmental failure of oligodendrocytes. $^{28}$ Thus, comprehensive studies investigating the long-term effects of deep hypothermia on WM protection are warranted.

\section{Study Limitations}

First, the brain slices were in a nonphysiologic state without cerebral flow perfusion and blood-brain barrier protection. The degree of the loss of brain WM is more severe than in the in vivo model. So this may underestimate the protective effects of minocycline in CPB and DHCA conditions in this study. Second, we have not demonstrated the direct cause and effect relationship between microglial activation and preOL injury because of the limitations of the brain slice model. Third, the prolonged roles of microglial activation in preOL loss cannot be observed in our model. Moreover, we did not study the dose-effect relationship of minocycline and its side effects on systemic organs in this brain slice model. Thus, the efficacy and safety of this drug should be carefully evaluated in a preclinical trial. In addition, abnormal brain development begins in utero. The resultant brain immaturity at birth is now thought to be a primary major risk factor underlying perioperative hypoxic/ischemic WM injury and subsequent neurodevelopmental disability after surgery for patients with CHD. This suggests that neuroprotective strategy initiated at the time of cardiac surgery as seen in this study is inadequate to improve the neurodevelopmental outcomes.

\section{CONCLUSIONS}

A neonatal rat brain slice model has been well established for replicating the WM injury induced by CPB and HCA. This study has demonstrated that microglial activation in WM plays a vital role in preOL injury. We have demonstrated that minocycline can inhibit the microglial activation, which may reduce preOL injury. This may potentially become a novel and feasible strategy for improving neuroprotection after neonatal cardiac surgery.

\section{Conflict of Interest Statement}

Authors have nothing to disclose with regard to commercial support.

The authors thank Nobuyuki Ishibashi for his assistance with the revised manuscript.

\section{References}

1. Mahle WT, Tavani F, Zimmerman RA, Nicolson SC, Galli KK, Gaynor JW, et al An MRI study of neurological injury before and after congenital heart surgery Circulation. 2002;106:1109-14.

2. Galli KK, Zimmerman RA, Jarvik GP, Wernovsky G, Kuypers MK, Clancy RR, et al. Periventricular leukomalacia is common after neonatal cardiac surgery. $J$ Thorac Cardiovasc Surg. 2004;127:692-704.

3. Beca J, Gunn JK, Coleman L, Hope A, Reed PW, Hunt RW, et al. New WM brain injury after infant heart surgery is associated with diagnostic group and the use of circulatory arrest. Circulation. 2013;127:971-9.

4. Back SA, Luo NL, Borenstein NS, Levine JM, Volpe JJ, Kinney HC. Late oligodendrocyte progenitors coincide with the developmental window of vulnerability for human perinatal WM injury. J Neurosci. 2001;21:1302-12.

5. Volpe JJ, Kinney HC, Jensen FE, Rosenberg PA. The developing oligodendrocyte: key cellular target in brain injury in the premature infant. Int J Dev Neurosci. 2011;29:423-40.

6. Ishibashi N, Scafidi J, Murata A, Korotcova L, Zurakowski D, Gallo V, et al. WM protection in congenital heart surgery. Circulation. 2012;125:859-71.

7. Lehnardt S. Innate immunity and neuroinflammation in the CNS: the role of microglia in Toll-like receptor-mediated neuronal injury. Glia. 2010;58:253-63.

8. He YF, Chen HJ, Qian LH, He LF, Buzby JS. Diphenyleneiodonium protects preoligodendrocytes against endotoxin-activated microglial NADPH oxidasegenerated peroxynitrite in a neonatal rat model of periventricular leukomalacia. Brain Res. 2013;1492:108-21.

9. Korotcova L, Kumar S, Agematsu K, Morton PD, Jonas RA, Ishibashi N. Prolonged white matter inflammation after cardiopulmonary bypass and circulatory arrest in a juvenile porcine model. Ann Thorac Surg. 2015;100:1030-7.

10. Drabek T, Tisherman SA, Beuke L, Stezoski J, Janesko-Feldman K, LahoudRahme M, et al. Deep hypothermia attenuates microglial proliferation independent of neuronal death after prolonged cardiac arrest in rats. Anesth Analg. 2009 109:914-23.

11. Cai Z, Lin S, Fan LW, Pang Y, Rhodes PG. Minocycline alleviates hypoxicischemic injury to developing oligodendrocytes in the neonatal rat brain. Neuroscience. 2006;137:425-35.

12. Andropoulos DB, Hunter JV, Nelson DP, Stayer SA, Stark AR, McKenzie ED, et al. Brain immaturity is associated with brain injury before and after neonatal cardiac surgery with high-flow bypass and cerebral oxygenation monitoring. $J$ Thorac Cardiovasc Surg. 2010;139:543-56.

13. Craig A, Luo NL, Beardsley DJ, Wingate-Pearse N, Walker DW, Hohimer AR, et al. Quantitative analysis of perinatal rodent oligodendrocyte lineage progression and its correlation with human. Exp Neurol. 2003;181:231-40.

14. Agematsu K, Korotcova L, Scafidi J, Gallo V, Jonas RA, Ishibashi N. Effects of preoperative hypoxia on white matter injury associated with cardiopulmonary bypass in a rodent hypoxic and brain slice model. Pediatr Res. 2014;75:618-25.

15. Schmitz T, Endesfelder S, Chew LJ, Zaak I, Buhrer C. Minocycline protects oligodendroglial precursor cells against injury caused by oxygen-glucose deprivation. J Neurosci Res. 2012;90:933-44.

16. Murata A, Agematsu K, Korotcova L, Gallo V, Jonas RA, Ishibashi N. Roden brain slice model for the study of white matter injury. J Thorac Cardiovasc Surg. 2013;146:1526-33.

17. Pena F. Organotypic cultures as tool to test long-term effects of chemicals on the nervous system. Curr Med Chem. 2010;17:987-1001.

18. Agematsu K, Korotcova L, Morton PD, Gallo V, Jonas RA, Ishibashi N. Hypoxia diminishes the protective function of white-matter astrocytes in the developing brain. J Thorac Cardiovasc Surg. 2016;151:265-72.

19. Asahi M, Wang X, Mori T, Sumii T, Jung JC, Moskowitz MA, et al. Effects of matrix metalloproteinase-9 gene knock-out on the proteolysis of blood-brain barrier and white matter components after cerebral ischemia. J Neurosci. 2001;21: 7724-32.

20. Liu MC, Akle V, Zheng W, Kitlen J, O'Steen B, Larner SF, et al. Extensive degradation of myelin basic protein isoforms by calpain following traumatic brain injury. J Neurochem. 2006;98:700-12. 
21. Liu XB, Shen Y, Plane JM, Deng W. Vulnerability of premyelinating oligodendrocytes to white-matter damage in neonatal brain injury. Neurosci Bull. 2013; 29:229-38.

22. Back SA, Han BH, Luo NL, Chricton CA, Xanthoudakis S, Tam J, et al. Selective vulnerability of late oligodendrocyte progenitors to hypoxia-ischemia. J Neurosci. 2002;22:455-63.

23. Dheen ST, Kaur C, Ling EA. Microglial activation and its implications in the brain diseases. Curr Med Chem. 2007;14:1189-97.

24. Weinstein JR, Koerner IP, Moller T. Microglia in ischemic brain injury. Future Neuron. 2010;5:227-46.

25. Gao HM, Hong JS. Why neurodegenerative diseases are progressive: uncontrolled inflammation drives disease progression. Trends Immunol. 2008;29: $357-65$.
26. Buller KM, Carty ML, Reinebrant HE, Wixey JA. Minocycline: a neuroprotective agent for hypoxic-ischemic brain injury in the neonate? J Neurosci Res. 2009;87:599-608.

27. Pokoma P, Wildschut ED, Vobruba V, van den Anker JN, Tibboel D. The impact of hypothermia on the pharmacokinetics of drugs used in neonates and young infants. Curr Pharm Des. 2015;21:5705-24.

28. Kanagawa T, Fukuda H, Tsubouchi H, Komoto Y, Hayashi S, Fukui O, et al. A decrease of cell proliferation by hypothermia in the hippocampus of the neonatal rat. Brain Res. 2006;1111:36-40.

Key Words: congenital heart defect, white-matter, microglia, minocycline, preoligodendrocyte

Readers who found these articles interesting may also like to read the following papers found in recent and future issues of our sister publications, Seminars in Thoracic and Cardiovascular Surgery and Operative Techniques in Thoracic and Cardiovascular Surgery!

\section{Congenital: Basic Science}

ORIGINAL SUBMISSION: Calcification or Not. This Is the Question. A 1-Year Study of Bovine Pericardial Vascular Patches (CardioCel) in Minipigs. Aida Salameh. Semin Thoracic Surg 2018: 54-59.

Editorial Commentary: Is This a Good Study? Christian Brizard. Semin Thoracic Surg 2018: 60-61. 